Revista

Ibero-Americana

de Estratégia

\title{
VANTAGEM COMPETITIVA: OPERACIONALIZANDO O CONCEITO A PARTIR DA RESOURCE-ADVANTAGE THEORY
}

COMPETITIVE ADVANTAGE: OPERATIONALIZING THE CONCEPT BASED ON THE RESOURCE-ADVANTAGE THEORY

\section{Ricardo Messias Rossi}

Pesquisador Sênior Centro de Pesquisa e Projetos em Marketing e Estratégia - MARKESTRAT

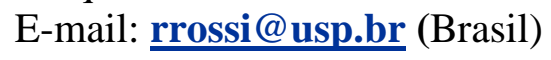

\author{
Andrea Lago Silva \\ Professora de Marketing no Departamento de Engenharia de Produção (DEP) da Universidade \\ Federal de São Carlos - UFSCar \\ E-mail: deialago@power.ufscar.br (Brasil)
}

Revista Ibero-Americana de Estratégia - RIAE, São Paulo, v. 8, n. 2, p. 31-57, jul./dez. 2009. 
Vantagem Competitiva: Operacionalizando o Conceito a partir da Resource-Advantage Theory

\title{
VANTAGEM COMPETITIVA: OPERACIONALIZANDO O CONCEITO A PARTIR DA RESOURCE-ADVANTAGE THEORY
}

\section{RESUMO}

O objetivo deste estudo foi desenvolver um método, a partir do conceito de vantagem competitiva, para análise interna de empresas inseridas em um contexto de competição de mercado. Foi realizada uma pesquisa exploratória e com abordagem qualitativa. A partir da revisão teórica da literatura pertinente ao tema, construiu-se um método preliminar de análise interna, posteriormente, aprimorado por meio das informações coletadas na pesquisa de campo. A pesquisa de campo foi composta por seis estudos de caso com empresas da indústria de defensivos agrícolas no Brasil. Como resultado, o estudo propõe uma forma de operacionalização do conceito de recursos (lacuna observada na teoria por alguns autores). Gerencialmente, o estudo contribui fornecendo aos administradores um método de análise que suporta as decisões referentes ao gerenciamento de recursos da organização.

Palavras-chave: Vantagem Competitiva; Recursos; Estratégia; Competição; Valor.

\section{COMPETITIVE ADVANTAGE: OPERATIONALIZING THE CONCEPT BASED ON THE RESOURCE-ADVANTAGE THEORY}

\begin{abstract}
The objective of this study was to develop a method based on the "competitive advantage" concept for the internal analysis of organizations in the context of competition. A qualitative and exploratory research was carried out. From a theoretical literature review on this subject, a preliminary internal analysis methodology was developed, which was then improved with the information collected from the field research. The field research consisted of six case studies conducted in companies working in the area of agrochemical industry in Brazil. Hence, this study proposes a way to operationalize the concept of resources (a theoretical gap observed by some authors). In managerial terms, this study contributes providing administrators with a method of analysis that deals with decisions regarding the management of resources of an organization.
\end{abstract}

Keywords: Competitive Advantage; Resources; Strategy; Competition; Value.

Revista Ibero-Americana de Estratégia - RIAE, São Paulo, v. 8, n. 2, p. 31-57, jul./dez. 2009. 


\section{INTRODUÇÃO}

Na década de 90, Hunt \& Morgan (1995) apresentaram um provocativo artigo sobre o modelo de competição, baseado nas vantagens comparativas em recursos das organizações. Este estudo seria novamente detalhado por esses autores (Hunt \& Morgan, 1996), culminando na proposição de um modelo geral de competição, denominado Resource-Advantage Theory (RA Theory). Esse modelo contestava algumas premissas dominantes no mainstream da Economia Neoclássica e, conseqüentemente, desencadeou um interessante debate entre pesquisadores de diversas correntes teóricas.

Em resumo, o modelo da RA Theory propõe que a competição é uma constante disputa entre firmas pela obtenção de vantagens comparativas em recursos que propiciarão uma posição de vantagem competitiva no mercado e, conseqüentemente, desempenho financeiro superior (Hunt, 2000a). Dessa forma, a estrutura da RA Theory mostra uma cadeia de relações entre causas e efeitos, onde a posição de vantagem competitiva de uma firma no mercado é a conseqüência das vantagens comparativas em recursos dessa firma e a causa de seu desempenho financeiro superior em relação aos competidores diretos.

Ao inserir o conceito de recursos dentro de uma dinâmica de competição, Hunt \& Morgan (1995), mostraram o caminho para ampliar e operacionalizar o conhecimento em relação aos recursos das organizações e a posição de vantagem competitiva. A relação entre o gerenciamento de recursos de uma firma e a obtenção de uma posição de vantagem competitiva tinha sido previamente estudada por Peteraf (1993). De fato, a possibilidade de utilização prática desses conceitos foi intensamente questionada em diversos textos, como nos trabalhos de Foss (1997), Connor (2002), Klein (2002) e Gibbert et al (2006).

A preocupação na operacionalização do conceito teórico não é recente, Ford \& Mahieu (1998) já indicavam que a Resource-Based View (RBV) avançava muito mais no campo teórico do que no aplicado. Littler \& Tynan (2005) indicam a importância de aumentar a publicação de artigos enfatizando as implicações gerenciais das teorias de marketing. Addis \& Podesta (2005) ressaltam a necessidade de diminuir a distância entre o conhecimento teórico e o aplicado.

Todavia, a operacionalização do conhecimento não é uma atividade simples. Ardley (2008) faz uma interessante reflexão sobre os equívocos e as simplificações excessivas do conhecimento de marketing na tentativa de representar a realidade.

Assim, a evolução do conhecimento em estratégia deve avançar simultaneamente no campo teórico e aplicado, sendo dever dos pesquisadores gerarem tanto teorias conectadas com a realidade

Revista Ibero-Americana de Estratégia - RIAE, São Paulo, v. 8, n. 2, p. 31-57, jul./dez. 2009. 
Vantagem Competitiva: Operacionalizando o Conceito a partir da Resource-Advantage Theory

quanto frameworks com sólida fundamentação teórica e não somente simplificações inadequadas. Esse trabalho propõe um framework gerado a partir da teoria e aprimorado por meio de uma pesquisa de campo com executivos de empresas.

O principal objetivo desse artigo é apresentar uma estrutura para análise interna das organizações. Esse framework é baseado na estrutura de competição proposta pela RA Theory (Hunt, 2000a) e visa servir como ferramenta de análise e suporte para a definição de estratégias competitivas das organizações. Primeiro será discutido o conceito de vantagem competitiva, bem como o modelo de competição proposto pela RA Theory. Ressalta-se que a estrutura inicial do framework proposto foi desenvolvida por meio da combinação de conhecimentos teóricos relacionados com estratégia e competição.

\section{VANTAGEM COMPETITIVA}

Conforme observado por Castro \& Chrisman (1995), a obtenção de uma posição de vantagem competitiva é essencial para as empresas no escopo da estratégia competitiva. Hunt (2000a) propõe que a vantagem competitiva só faz sentido em um contexto de competição. Ela é conseqüência de uma vantagem em recursos e causa de desempenho financeiro superior. Klein (2002) discute que embora o termo "vantagem competitiva" seja muito estudado e citado, ainda existe confusão sobre seu significado e emprego.

Esse termo tornou-se popular após Porter (1985) publicar o livro Competitive Advantage. No entanto, o trabalho considerado precursor do conceito foi o artigo de Alderson (1937) que propõe bases para a obtenção de vantagens diferenciais. Hall (1980), por sua vez, insere a idéia de que as organizações obtêm sucesso por meio de vantagens em custo ou diferenciação, idéia que foi mais bem explorada por Porter (1980). Henderson (1983) discute a importância de uma empresa possuir uma "vantagem única" em relação aos concorrentes para sobressair em um ambiente competitivo. Day (1984) insere a idéia de "vantagem competitiva sustentável". Embora Porter (1985) tenha discutido a sustentação da posição de vantagem competitiva, Coyne (1986) aprofundou as bases de sustentação da vantagem competitiva, obtida por meio da criação de um gap entre a empresa e seus competidores. No mesmo ano, Ghemawat (1986) também discutiu as bases de sustentação da vantagem competitiva.

Day \& Wensley (1988) aprofundam os estudos sobre as fontes de vantagem competitiva e as formas de sustentação dessas vantagens. A dinâmica de geração de vantagem competitiva 
apresentada por esses autores é a base da estrutura proposta na RA Theory. Day \& Wensley (1988) indicam que as fontes de vantagem de uma empresa são seus recursos e habilidades superiores, que lhe permitirão desfrutar de posições de vantagem por meio da entrega de valor relativo superior ou custos relativos inferiores, que, por sua vez, resultarão em desempenhos satisfatórios (satisfação, lealdade, participação de mercado e lucratividade).

Para Hamel \& Prahalad (1989), em um contexto competitivo, as firmas não devem focalizar seus esforços para copiar a vantagem de seus concorrentes, ao contrário, devem implementar uma cultura e visão de longo-prazo visando desenvolver uma nova forma de vantagem, baseada em inovação.

Peteraf (1993) propõe que a vantagem competitiva sustentável de uma empresa está baseada em quatro condições: (1) heterogeneidade de recursos entre firmas; (2) limitação da competição expost; (3) mobilidade imperfeita dos recursos; (4) limitação da competição ex-ante. Para a autora, a heterogeneidade dos recursos explica a obtenção de retornos financeiros superiores em relação aos concorrentes, ou seja, uma posição de vantagem competitiva. Essa posição será sustentável se existirem limitações da competição ex ante (uma barreira de entrada como a necessidade de capital, por exemplo), limitações da competição ex-post (dificuldade de imitação e de substituição dos recursos) e mobilidade imperfeita dos recursos (impossibilidade de transferir recursos de uma firma para outra).

Rowe \& Barnes (1998) e Weerawardena \& O’Cass (2004) mostram que empresas dirigidas ao mercado têm maior possibilidade de obter vantagens competitivas sustentáveis. Wright et al. (1995) concluíram que a orientação da empresa interfere na obtenção de vantagem competitiva. Olusoga, Mokwa \& Noble (1995) inserem uma importante discussão ao relacionar os conceitos de grupos estratégicos e de barreiras à mobilidade e à obtenção de vantagem competitiva. Nesse estudo, os autores concluíram que a análise de grupos estratégicos pode ser útil para identificar as firmas que obtiveram vantagem competitiva em uma indústria. Rouse \& Daellenbach (1999) também consideraram importante a análise de grupos estratégicos para o entendimento da vantagem competitiva. Castro \& Chrisman (1995) relacionaram a ordem de entrada no mercado e a estratégia competitiva com o desempenho das empresas. Esses autores concluíram que tanto a ordem de entrada no mercado (pioneirismo) quanto as opções estratégicas da empresa interferem na manutenção de uma posição de vantagem competitiva.

Oliver (1997) acrescenta que, além dos recursos, fatores institucionais (nos níveis de análise individual da organização ou da indústria) também interferem na obtenção de vantagem competitiva sustentável. Para Rindova \& Fombrun (1999), além dos fatores econômicos, fatores sociocognitivos

Revista Ibero-Americana de Estratégia - RIAE, São Paulo, v. 8, n. 2, p. 31-57, jul./dez. 2009. 
Vantagem Competitiva: Operacionalizando o Conceito a partir da Resource-Advantage Theory

(reputação, medidas de sucesso, conhecimento, crenças, identidade, entre outros) ajudam a explicar as vantagens competitivas da firma.

Ma (1999a) desenvolveu uma estrutura denominada SELECT (Substance; Expression; Locale; Effect; Cause; Time-Span) para analisar a "anatomia" da vantagem competitiva. Com o intuito de servir como guia prático para executivos, essa estrutura de análise caracteriza a vantagem competitiva em função de seis grupos de atributos. Em outro artigo, Ma (1999b) propõe três fontes genéricas de vantagem competitiva: (1) baseada na propriedade; (2) baseada no conhecimento; (3) baseada no acesso.

Chaharbaghi \& Lynch (1999) inserem o conceito de vantagem estratégica. O termo vantagem estratégica refere-se à dinâmica da organização utilizada para desenvolver recursos estratégicos garantindo a constante renovação desses recursos. Em outras palavras, a vantagem estratégica é a habilidade da empresa em desenvolver constantemente seus recursos estratégicos visando garantir uma vantagem ao longo do tempo em relação a seus competidores. Lei \& Slocum (2002) consideram que a renovação de recursos estratégicos é uma atividade fundamental para sustentar uma posição de vantagem competitiva em um ambiente que muda cada vez mais rápido. Para Fahy (1999), a principal função dos administradores é identificar, desenvolver, proteger e desdobrar os recursos da empresa.

Klein (2002) indica que equiparar vantagem competitiva com o benefício gerado por ela, que para vários autores é o desempenho financeiro superior, torna o termo tautológico e circular, pois significaria dizer que "desempenho financeiro superior é a fonte de desempenho financeiro superior". Para evitar esse equívoco, deve-se considerar que a vantagem competitiva é uma condição da empresa em determinado mercado, e a conseqüência dessa posição é o desempenho financeiro superior, conforme proposto por Hunt (2000a). Assim, vantagem competitiva deve ser definida em termos de geração de valor superior, que é sua causa, e não em termos de desempenho financeiro superior, que é sua conseqüência. Besanko, Dranove \& Shanley (2000) argumentam que a observação de desempenho financeiro superior é uma indicação de uma posição de vantagem competitiva (mas o desempenho em si não é a vantagem).

Christensen (2001) discute que os direcionadores de vantagem competitiva dependem não só das características da empresa, mas também das características da indústria. O autor posiciona algumas indústrias em diferentes momentos históricos e, dentro de cada contexto específico, indica a razão da predominância de diferentes direcionadores, tais como economia de escala, economia de escopo, integração vertical e outsourcing, e competências.

Revista Ibero-Americana de Estratégia - RIAE, São Paulo, v. 8, n. 2, p. 31-57, jul./dez. 2009. 
Tanto Bartlett \& Ghoshal (2002) quanto Lubit (2001) consideram o gerenciamento do conhecimento como a maior fonte potencial para geração de vantagem competitiva sustentável. Cardy \& Selvarajan (2006) consideram que as competências individuais e organizacionais são importantes para criação de valor e, portanto, devem ser identificadas.

Kothandaraman \& Wilson (2001) consideram que a competição cada vez mais será entre redes de empresas do que entre empresas isoladamente. Portanto, os relacionamentos entre empresas que geram valor para o cliente cada vez mais serão fonte de vantagem competitiva. Hunt (2000a) considera que os "recursos relacionais" são a fonte desse tipo de geração de valor. Cousins (2005) conclui que a estratégica genérica da empresa influencia a gestão da cadeia de suprimentos. Segundo esse autor, empresas com foco em custo geralmente consideram o gerenciamento da cadeia de suprimentos como uma atividade de redução de custos; por outro lado, empresas com foco em diferenciação buscam gerenciar a cadeia de suprimentos com intuito torná-la uma capacidade distinta.

Miles \& Darroch (2006) consideram que a vantagem competitiva segue um ciclo de descoberta/criação e dissipação. A dissipação de uma posição de vantagem competitiva ocorre devido ao processo de competição. Segundo Lieberman \& Asaba (2006), as firmas imitam umas às outras por dois motivos: (1) quando percebem que a outra firma possui mais informação e (2) para tentar manter uma paridade competitiva ou limitar a rivalidade. Hunt (2000a) propõe que o mecanismo que indica uma posição de vantagem ou desvantagem competitiva é o desempenho financeiro. Assim, as firmas aprendem com seu desempenho e, caso esse seja inferior a um concorrente, tentarão reagir copiando o concorrente ou realizando uma inovação. Dessa forma a competição gera desequilíbrio e, por esse motivo, uma empresa detentora de uma posição de vantagem competitiva precisa constantemente investir na manutenção dessa posição (Miles \& Darroch 2006). Adner \& Zemsky (2006) propõem que, além da ação dos competidores, as mudanças de percepção dos consumidores podem erodir uma vantagem competitiva.

\section{RESOURCE-ADVANTAGE THEORY}

A partir do artigo publicado por Hunt \& Morgan (1995), a RA Theory foi discutida e aprimorada em uma seqüência de publicações (Hunt, 1995; Hunt, 1997a; Hunt, 1997b; Hunt, 1997c; Hunt, 1997d; Hunt, 1998; Hunt, 1999; Hunt, 2000b; Hunt, 2001; Hunt, 2002; Hunt \& Arnett, 2001; Hunt \& Arnett, 2004; Hunt \& Derozier, 2004; Hunt \& Duhan, 2002; Hunt \& Lambe,

Revista Ibero-Americana de Estratégia - RIAE, São Paulo, v. 8, n. 2, p. 31-57, jul./dez. 2009. 
Vantagem Competitiva: Operacionalizando o Conceito a partir da Resource-Advantage Theory

2000; Hunt \& Morgan, 1996; Hunt \& Morgan, 1997; Morgan \& Hunt, 2002). No entanto, a descrição mais detalhada dessa teoria é encontrada no livro A general theory of competition: resources, competences, productivity, economic growth (Hunt, 2000a).

Segundo a RA Theory, a competição é uma constante luta pela obtenção de vantagens comparativas em recursos que propiciarão uma posição de vantagem competitiva no mercado e, conseqüentemente, desempenho financeiro superior, conforme mostrado na Figura 1 (Hunt, 2001).

Figura 1 - Esquema da Resource-Advantage Theory.

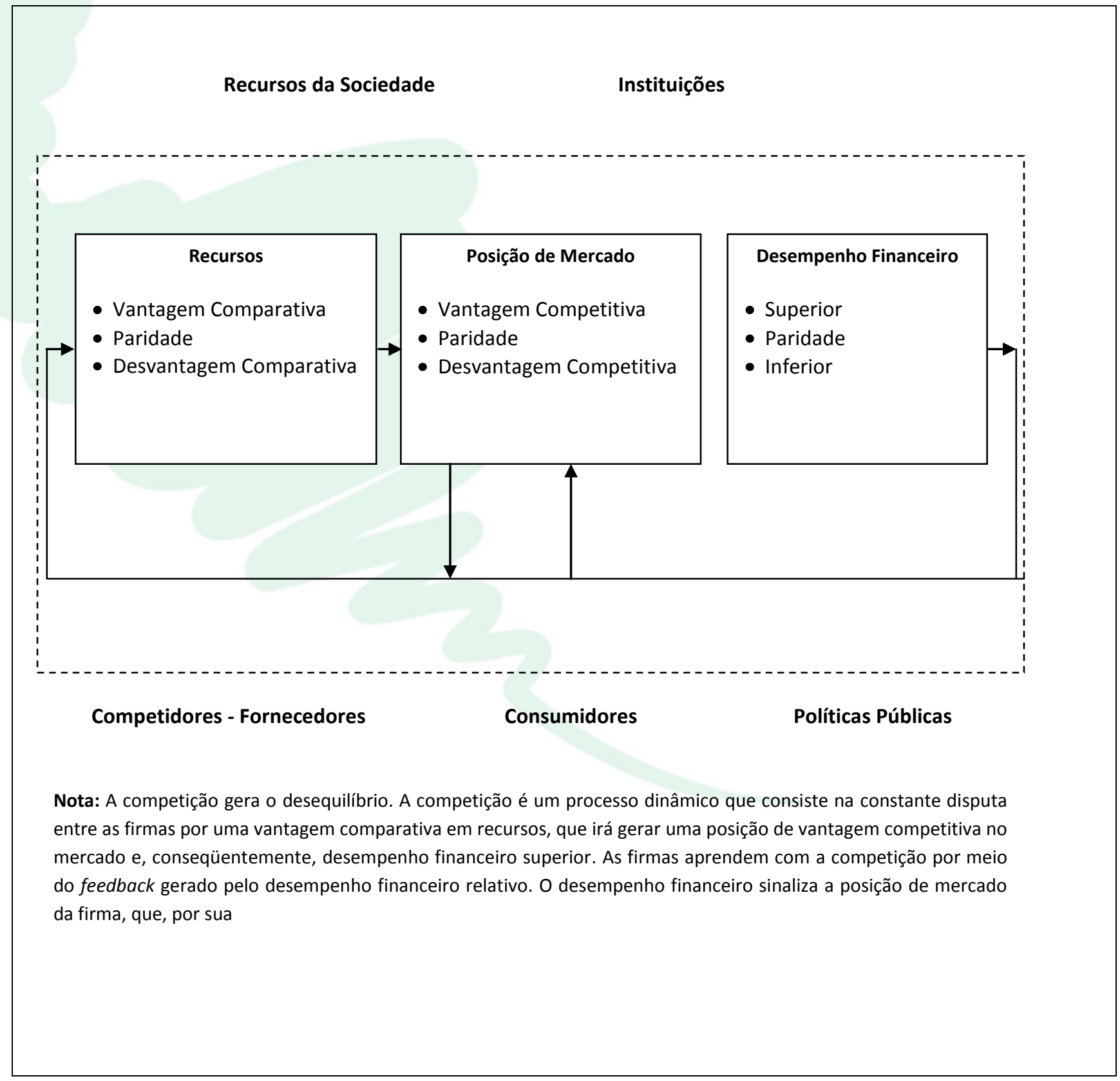

Revista Ibero-Americana de Estratégia - RIAE, São Paulo, v. 8, n. 2, p. 31-57, jul./dez. 2009. 
Posições de vantagem (ou desvantagem) competitiva resultarão em desempenho financeiro superior (ou inferior). As firmas podem obter uma posição de vantagem competitiva quando possuem uma gama de recursos que permitam entregar para um determinado segmento de mercado uma oferta de (a) valor superior percebido e/ou (b) produzida com custos mais baixos (Hunt \& Morgan, 1995). Esse processo de disputa por posições de vantagem competitiva é influenciado por variáveis externas, especialmente as políticas públicas, os consumidores, os fornecedores e competidores, os recursos da sociedade e as instituições. Assim, segundo esses autores, nove posições de mercado são possíveis (Figura 2).

Figura 2 - Matriz das Posições Competitivas.

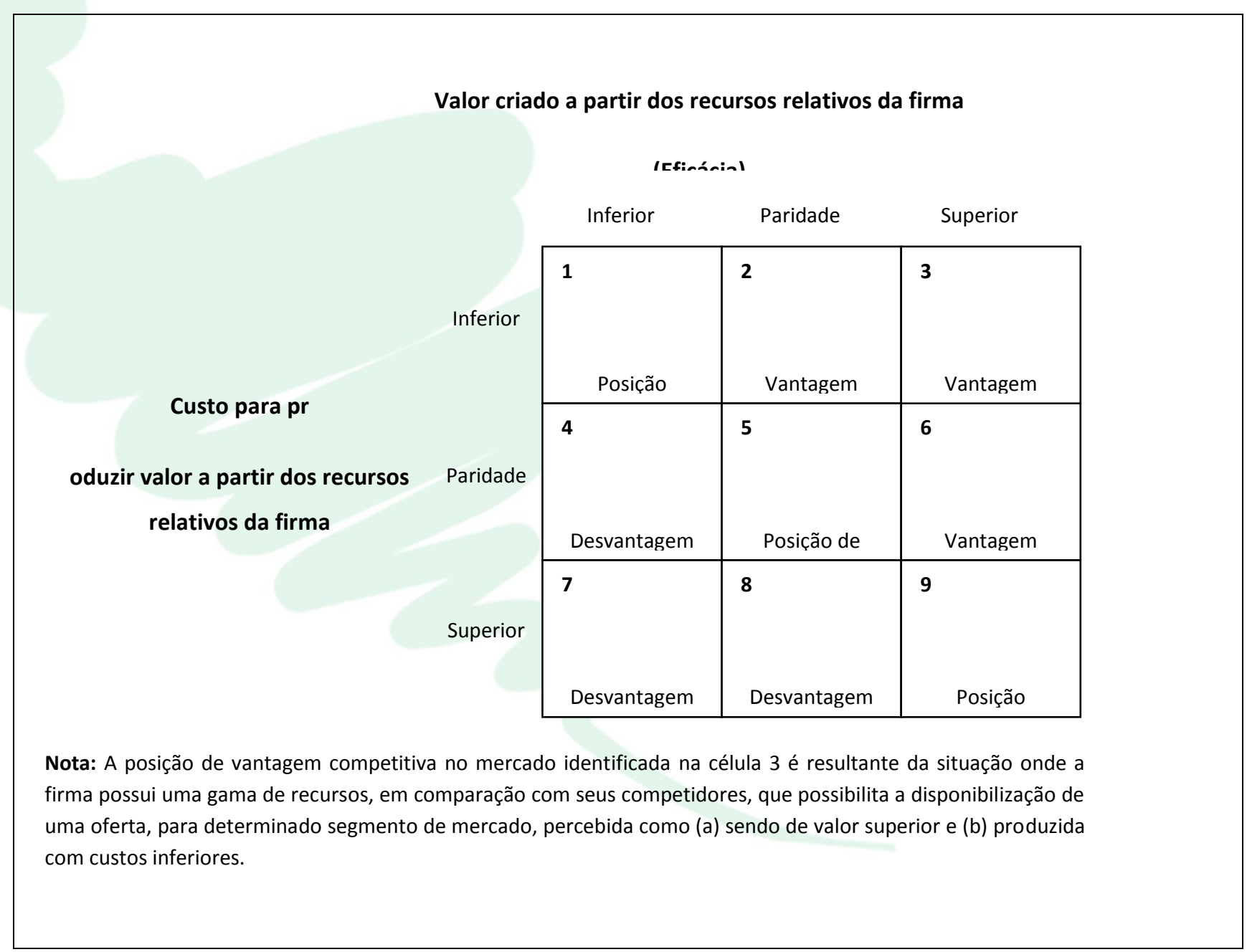

Fonte: Hunt (2001, p. 529).

Revista Ibero-Americana de Estratégia - RIAE, São Paulo, v. 8, n. 2, p. 31-57, jul./dez. 2009. 
Vantagem Competitiva: Operacionalizando o Conceito a partir da Resource-Advantage Theory

As células de números 2, 3 e 6 indicam posições de vantagem competitiva. A célula 3 indica uma posição competitiva ideal para uma firma, em que as vantagens comparativas em recursos conseguem produzir valor superior com menos custo. As células 2 e 6 indicam respectivamente uma vantagem em custo e em geração de valor. Já as células de números 4, 7 e 8 indicam posições de desvantagem competitiva, demonstrando a inabilidade da firma na combinação de recursos, o que, conseqüentemente, resultará em retornos inferiores. As posições indicadas nas células 1 e 9 correspondem a uma situação indeterminada, em que a vantagem comparativa, em valor ou custo, pode gerar retornos superiores, similares (paridade) ou inferiores. Por último, a célula 5 corresponde a uma posição de paridade, sendo a situação de mercado explicada em parte pela Teoria da Competição Perfeita.

A sustentabilidade da situação de vantagem comparativa em recursos é determinada por fatores internos e externos. Internamente, Hunt (2000a) propõe que as empresas podem perder essa posição de vantagem (1) se falharem em reinvestir na manutenção dos recursos, (2) na presença de ambigüidade causal que dificulte para firma compreender a importância de seus recursos e (3) na falha de adaptação às mudanças.

Externamente, a vantagem comparativa em recursos que uma firma possui pode ser neutralizada pelas mudanças nos recursos da sociedade e nas instituições e pelas ações de consumidores, governo, fornecedores e competidores (Hunt, 2000a). Para Barney (1991) e Reed \& DeFillippi (1990), as ações que os competidores podem realizar para neutralizar a vantagem comparativa em recursos de uma firma incluem a compra de recursos iguais, a imitação desses recursos e o desenvolvimento de recursos estratégicos equivalentes ou superiores. Esses autores concordam que a sustentação de uma situação de vantagem comparativa em recursos depende da capacidade de a firma gerar barreiras à imitação. Morgan, Vorhies \& Schlegelmilch (2006) encontraram evidências de que são importantes direcionadores de desempenho as barreiras à imitação criadas por uma firma e a inabilidade dos competidores em usarem recursos substitutos para neutralizar uma vantagem comparativa.

A RA Theory é coerente com os conceitos de aprendizado organizacional, ou seja, no decorrer do tempo, as firmas aprendem de várias formas. A RA Theory amplia o conhecimento nessa área por meio da discussão de como o processo de competição contribui para o aprendizado organizacional. O feedback gerado pelo processo de competição é referente ao desempenho financeiro relativo, por meio do qual as firmas reconhecem (ou pensam reconhecer) seus recursos relativos e suas posições no mercado (Hunt, 2000a). Assim, competição é um processo de

Revista Ibero-Americana de Estratégia - RIAE, São Paulo, v. 8, n. 2, p. 31-57, jul./dez. 2009. 
conhecimento e descoberta (Hayek, 1945). Em outras palavras, as firmas aprendem competindo, sendo esse um processo evolucionário.

\section{PESQUISA DE CAMPO}

A pesquisa apresentada neste artigo é exploratória e aplicada, com abordagem qualitativa. $\mathrm{O}$ estudo foi dividido em quatro fases: (1) revisão teórica; (2) elaboração do método preliminar de análise interna; (3) pesquisa de campo; (4) elaboração final do método de análise interna.

A primeira etapa da pesquisa foi a combinação teórica dos conceitos de recursos e vantagem competitiva, utilizado para isso o modelo de competição proposto na RA Theory. Em seguida, foi gerado preliminarmente um framework para a análise interna das organizações. Na terceira etapa da pesquisa, esse framework preliminar foi submetido à avaliação de executivos das empresas analisadas nos estudos de caso. Esses executivos analisaram, criticaram e validaram (em termos de viabilidade e relevância) o framework proposto. A partir de suas críticas, o framework preliminar foi aperfeiçoado e, assim, o framework final proposto neste artigo foi estruturado.

A pesquisa de campo foi realizada com empresas da indústria de defensivos agrícolas no Brasil. Esta indústria foi escolhida por ser altamente competitiva e possuir empresas que adotam posições estratégicas bem definidas, o que facilita a identificação de grupos estratégicos específicos. Seguindo a tipologia proposta por Bryman (2005), esta pesquisa de campo foi uma pesquisa qualitativa organizacional, baseada em entrevistas. Foi utilizada a técnica de entrevista em profundidade, em que os entrevistados são escolhidos por terem algum conhecimento especializado (Hair Jr. et al 2003).

Inicialmente foi caracterizado o setor de defensivos agrícolas no Brasil. Em seguida foram identificados e caracterizados os dois principais grupos estratégicos de empresas que constituem esse setor (genéricos e especialidades). Na seqüência, foram selecionadas seis empresas para a realização dos estudos de caso. Para as empresas selecionadas, foram levantados dados secundários (material institucional) para caracterizar cada empresa em termos de: histórico e origem; unidades de negócios, linhas de produtos, mercados-alvo e relatórios financeiros. Por último, foram identificados os executivos que deveriam ser entrevistados. Foram selecionados executivos envolvidos nas decisões estratégicas competitivas da unidade de negócio de defensivos agrícolas. Esses executivos possuíam diferentes cargos dentro da alta administração das organizações, possuindo em comum o fato de estarem envolvidos diretamente com atividades de administração

Revista Ibero-Americana de Estratégia - RIAE, São Paulo, v. 8, n. 2, p. 31-57, jul./dez. 2009. 
Vantagem Competitiva: Operacionalizando o Conceito a partir da Resource-Advantage Theory

estratégica. Foram entrevistados executivos com cargos de CEO, CFO, BMI Manager, Key Account Manager, Marketing Channels Manager e Strategic Marketing Manager.

O objetivo das entrevistas foi aprimorar o framework de análise interna proposto. Para auxiliar na entrevista, foi elaborado um roteiro semi-estruturado com os tópicos que deveriam ser discutidos. As entrevistas foram gravadas e ocorreram nos meses de agosto e setembro de 2008. As entrevistas tiveram uma duração média de 2 horas e trinta minutos. A estratégia analítica dos casos foi baseada nas proposições teóricas do método de análise interna, conforme classificação proposta por Yin (2002). A técnica analítica utilizada foi a criação de uma matriz de categorias e a disposição das evidências coletadas dentro dessas categorias (Yin, 2002). A Figura 3 resume os procedimentos da pesquisa de campo.

Figura 3 - Os procedimentos da pesquisa de campo.

\begin{tabular}{|c|c|}
\hline \multicolumn{2}{|c|}{ MÉTODO DE ESTUDO DE CASO } \\
\hline Propósito do estudo de caso & Exploratório. \\
\hline Objetivo do estudo de caso & Aprimorar o método de análise interna proposto \\
\hline Indústria analisada & Defensivos Agrícolas. \\
\hline Empresas estudadas & Monsanto; Syngenta; BASF; Ouro Fino; Produquímica; SipCam \\
\hline Unidade geográfica & Brasil. \\
\hline Grupos estratégicos identificados & Dois (especialidades e genéricos) \\
\hline Número de casos estudados & Seis (casos múltiplos) \\
\hline Coleta de dados secundários & Material Institucional (sites, catálogos e relatórios financeiros). \\
\hline Coleta de dados primários & Entrevistas em profundidade. \\
\hline Respondentes & Executivos envolvidos na elaboração de estratégias competitivas. \\
\hline Instrumento de apoio & Roteiro semi-estruturado \\
\hline
\end{tabular}

Revista Ibero-Americana de Estratégia - RIAE, São Paulo, v. 8, n. 2, p. 31-57, jul./dez. 2009. 


\section{MÉTODO DE ESTUDO DE CASO}

Período de realização das entrevistas

Fonte: Elaborado pelos autores.

Nos estudos de caso o processo de preparação e a realização das entrevistas seguiram o roteiro proposto por Voss, Tsikriktsis \& Frohlich (2002). Foi realizado um contato telefônico para apresentação e, depois que o executivo aceitou participar da pesquisa, foi enviada por e-mail uma carta-convite explicando o trabalho, esclarecendo os procedimentos de pesquisa e confirmando o agendamento. Após a confirmação do agendamento (local, data e horário da entrevista), foi enviada por e-mail uma síntese executiva apresentando a versão preliminar do método de análise interna proposto (framework), mostrando os templates sugeridos para realização da análise e um exemplo de preenchimento. Os pesquisadores estudaram previamente as características da empresa com o intuito de compreender melhor as especificidades do negócio, facilitando assim a discussão.

Ao término dos estudos de caso, o framework de análise interna proposto no início do processo foi modificado em função da interpretação que os pesquisadores fizeram em relação à opinião dos respondentes. Esse método modificado (aprimorado) é o método final de análise interna da empresa, baseado no conceito de recursos.

\section{FRAMEWORK PARA ANÁLISE INTERNA DAS ORGANIZAÇÕES}

O framework para análise interna das organizações, proposto nesse artigo, é constituído por seis etapas. Cada etapa representa um conjunto de atividades que operacionalizam o processo de análise interna das organizações. A Figura 4 mostra a estrutura geral do método proposto.

Revista Ibero-Americana de Estratégia - RIAE, São Paulo, v. 8, n. 2, p. 31-57, jul./dez. 2009. 
Vantagem Competitiva: Operacionalizando o Conceito a partir da Resource-Advantage Theory

Figura 4 - Estrutura General do Método de Análise Proposto.

\section{Etapa 1: Identificação do objeto de análise}

Atividade 1.1: Comitê de Decisões Estratégicas Competitivas

Atividade 1.2: Objetivos Corporativos

Atividade 1.3: Determinação dos pares produto/mercado

> Atividade 1.4: Identificação de Grupos Estratégicos

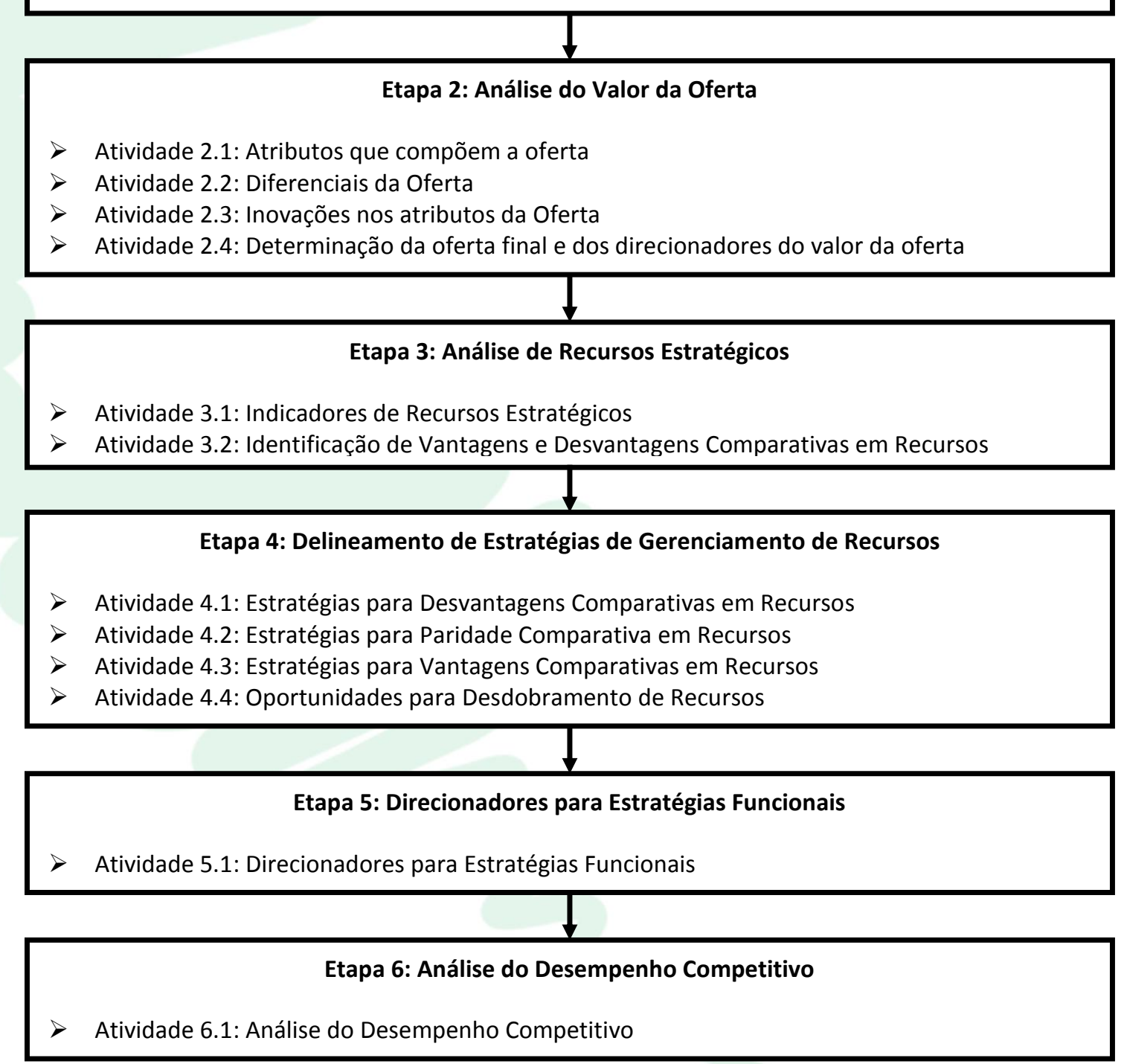

Fonte: Elaborado pelos autores.

Revista Ibero-Americana de Estratégia - RIAE, São Paulo, v. 8, n. 2, p. 31-57, jul./dez. 2009. 


\subsection{IDENTIFICAÇÃO DO OBJETO DE ANÁLISE}

A primeira etapa do framework é a "Identificação do objeto de análise" que visa preparar a organização para o início do processo de análise. Nessa fase ocorrerá a formação do comitê de decisões estratégicas. Também serão analisados os objetivos corporativos. Apesar de o framework ser destinado para a unidade de negócio, deve-se analisar a missão, a visão, os objetivos, os valores, os princípios estratégicos e as metas corporativas, para que toda a análise esteja baseada nessas diretrizes, evitando, assim, ações contraditórias dentro da organização.

A terceira atividade da primeira etapa do framework é a segmentação de mercados e de produtos. Os executivos podem considerar um único par produto/mercado ou vários pares de produtos/mercados. Quanto mais heterogêneo o mercado-alvo da organização, maior a necessidade de realizar a segmentação de mercado. Quanto maior a heterogeneidade de produtos/serviços, maior a necessidade de segmentação de produtos/serviços. Finalizando a primeira etapa do framework, deverá ser realizada a identificação dos grupos estratégicos relevantes que atuam em cada par de produto/mercado selecionado. Serão concorrentes diretos as empresas que compartilham o mesmo grupo estratégico. Quando as estratégias de todos os competidores são convergentes, pode existir um único grupo estratégico para o mercado analisado. A presença de mais de um grupo estratégico no mercado indica uma situação de competição direta (entre empresas do mesmo grupo estratégico) e indireta (entre as empresas de diferentes grupos estratégicos). A implicação estratégica da existência de mais de um grupo estratégico no mercado é a decisão de a organização procurar uma posição de destaque em seu grupo estratégico ou decidir pela migração para outro grupo estratégico.

\subsection{ANÁLISE DO VALOR DA OFERTA}

A segunda etapa do método visa analisar o valor da oferta da organização. Nesta fase, serão identificados os atributos que compõem o valor da oferta. Também serão identificados os diferenciais entre a oferta da organização e dos concorrentes diretos. Esta atividade mostrará os diferenciais, em termos de benefícios e custos, entre a oferta da organização e dos concorrentes, em dois sentidos: (a) os atributos distintos na oferta da organização em relação a seus competidores diretos e (b) os atributos distintos presentes nas ofertas dos competidores diretos em relação à oferta da organização.

Deve ser indicada, para cada atributo distinto, a decisão de manutenção ou não do diferencial. Essa decisão será baseada na relação entre os custos e benefícios associados à 
Vantagem Competitiva: Operacionalizando o Conceito a partir da Resource-Advantage Theory

manutenção de cada atributo. Em seguida, serão analisados os atributos diferenciais existentes nas ofertas dos concorrentes diretos em relação à oferta da organização. Serão analisados quais desses atributos deverão ser neutralizados, também com a análise baseada na relação entre custos e benefícios

O valor da oferta pode ser aumentado de três formas. A primeira é com o aumento de benefícios em uma proporção superior ao aumento de custos (eficácia). A segunda é com uma redução de custos em uma proporção maior do que a diminuição de benefícios (eficiência). A terceira é o aumento de benefícios com redução de custos (eficiência e eficácia).

A última atividade da segunda etapa framework é a determinação da oferta final e dos direcionadores de valor da oferta. Os direcionadores de valor da oferta serão a base para a análise de recursos, que é a próxima etapa do framework. Deverá ser definida a estratégia de proposição de valor que a organização adotará. Conforme tipologia proposta por Anderson, Narus \& Rossum (2006), três opções de estratégia de proposição de valor podem ser definidas: "todos os benefícios" (destacar todos os atributos que geram valor para a oferta); "pontos favoráveis de diferenciação" (destacar os atributos que diferenciam a oferta da organização em relação às ofertas dos concorrentes); "foco ressonante" (destacar os atributos principais que justificam para o cliente o valor superior da oferta da organização em relação às ofertas dos concorrentes).

\subsection{ANÁLISE DE RECURSOS ESTRATÉGICOS}

Uma vez identificados os direcionadores de valor, na terceira etapa do framework serão analisados os recursos estratégicos da organização. A primeira atividade desta etapa é a tradução dos direcionadores de valor em indicadores de recursos estratégicos. Serão identificadas as vantagens e desvantagens comparativas em recursos. Para sinalizar as posições de vantagem, paridade ou desvantagem comparativa, serão utilizados os indicadores de recursos estratégicos identificados na atividade anterior.

Cada indicador de recursos estratégicos será analisado em função de seu desempenho e custos relativos em relação aos concorrentes diretos. A partir desta análise, será possível identificar as posições de desvantagem comparativa, paridade ou vantagem comparativa em recursos. Complementarmente, será possível identificar se a posição é baseada em eficiência, eficácia ou ambas simultaneamente.

Revista Ibero-Americana de Estratégia - RIAE, São Paulo, v. 8, n. 2, p. 31-57, jul./dez. 2009. 


\subsection{DELINEAMENTO DE ESTRATÉGIAS DE GERENCIAMENTO DE RECURSOS}

A quarta etapa do método visa delinear estratégias para o gerenciamento de recursos da organização. A primeira atividade desta etapa é o delineamento de estratégias para neutralizar desvantagens comparativas. Esta atividade engloba a análise das opções estratégicas para neutralizar posições de desvantagens comparativas por meio do desenvolvimento de recursos. $\mathrm{O}$ objetivo da organização será neutralizar suas desvantagens. Para isso, são sugeridas duas estratégias básicas. Quando possível, neutralizar a desvantagem copiando as boas práticas do concorrente de melhor desempenho, por meio do benchmarking competitivo. Quando não é possível copiar o concorrente, devido à limitação de recursos (capital, por exemplo) ou à dificuldade de identificação da real fonte de vantagem do concorrente (ambigüidade causal, path dependencies ou complexidade social), a organização deve tentar uma inovação.

Também será realizada a análise das opções estratégicas para desenvolver recursos no caso da posição de paridade comparativa. A organização deve investir no desenvolvimento dos recursos em situação de paridade por dois motivos. Primeiro, para evitar que a paridade se transforme em desvantagem devido à evolução dos concorrentes. Segundo, para transformar a paridade em uma vantagem e, assim, destacar-se em relação aos concorrentes. A organização deve definir se pretende inovar para reduzir custos, aprimorar o desempenho ou ambos simultaneamente.

Em seguida será realizada a análise das opções estratégicas para proteger as posições de vantagens comparativas por meio do desenvolvimento de recursos. Duas estratégias básicas podem ser adotadas para as posições de vantagens comparativas em recursos. A primeira, uma vez identificado que a vantagem comparativa não é sustentável (pode ser copiada pelos concorrentes), é o desenvolvimento de inovações que tornem a combinação de recursos mais difícil de ser copiada. A segunda estratégia, para vantagens comparativas que dificilmente poderão ser copiadas pelos concorrentes, é o investimento contínuo para o desenvolvimento dos recursos que sustentam a vantagem, evitando a erosão dessa posição.

Por fim, serão analisadas as oportunidades para desdobramento de recursos. A última atividade da quarta etapa do método é a identificação de oportunidades para o desdobramento de recursos. Essa atividade visa identificar oportunidades de maximizar a utilização dos recursos existentes na organização, principalmente, aproveitando sinergias existentes.

Revista Ibero-Americana de Estratégia - RIAE, São Paulo, v. 8, n. 2, p. 31-57, jul./dez. 2009. 
Vantagem Competitiva: Operacionalizando o Conceito a partir da Resource-Advantage Theory

\subsection{DIRECIONADORES PARA ESTRATÉGIAS FUNCIONAIS}

Esta etapa do framework serve de ligação entre os níveis de estratégias competitivas e funcionais da organização. A análise interna da organização fornecerá os direcionadores para as estratégias funcionais. A partir desses direcionadores, planos de ação serão desenvolvidos e operacionalizados por meio de projetos específicos. Os direcionadores para estratégias funcionais são obtidos a partir das análises realizadas na quarta etapa do framework, para cada indicador analisado.

Para cada área funcional da organização deverão ser listados os indicadores analisados na quarta etapa do método. A partir da estratégia definida anteriormente para cada indicador, deverão ser listados os direcionadores de diferenciação e custo para as áreas funcionais da organização. A partir desses direcionadores, serão definidos os objetivos, os planos de ação, os projetos e as métricas de análise de desempenho para as áreas funcionais. O gerenciamento dessas atividades não está no escopo da estratégia competitiva, mas da estratégia funcional.

\subsection{ANÁLISE DO DESEMPENHO COMPETITIVO}

A última etapa do método de análise interna é destinada à elaboração de uma métrica para análise de desempenho competitivo. Seguindo o modelo da Resource-Advantage Theory, proposto por Hunt (2000a), o indicador da posição de vantagem competitiva é o desempenho financeiro superior. Assim, a métrica de análise de desempenho será dividida em duas partes. A primeira, os indicadores primários, com foco no desempenho financeiro e identificação da posição da empresa na matriz competitiva (desvantagem, paridade ou vantagem competitiva); a segunda, com foco nos indicadores de apoio, em função de outros objetivos da organização.

\section{CONCLUSÕES E IMPLICAÇÕES GERENCIAIS}

O framework proposto mostra uma forma de operacionalizar os conceitos de "recursos" e de "vantagem competitiva" para a realização da análise interna das organizações. Esse framework é constituído por cinco etapas:

Revista Ibero-Americana de Estratégia - RIAE, São Paulo, v. 8, n. 2, p. 31-57, jul./dez. 2009. 
- Etapa 1: Identificação do objeto de análise;

- Etapa 2: Análise do Valor da Oferta;

- Etapa 3: Análise de Recursos Estratégicos;

- Etapa 4: Delineamento de Estratégias de Gerenciamento de Recursos;

- Etapa 5: Direcionadores para Estratégias Funcionais;

- Etapa 6: Análise do Desempenho Competitivo.

A posição de vantagem competitiva deve ser analisada para cada par oferta/mercado da organização e em relação aos competidores de um mesmo grupo estratégico. Uma organização pode ter uma posição de vantagem competitiva em um determinado mercado-alvo e uma posição de desvantagem em outro. Além do mais, a posição de vantagem competitiva pode ser baseada em eficiência, eficácia ou em ambas simultaneamente.

O framework proposto contribui em relação aos modelos pré-existentes, pois: auxilia no posicionamento dentro de um grupo estratégico; na identificação dos atributos que geram valor para a oferta; na definição de modificações nas características da oferta; na identificação de desvantagens, paridades, vantagens comparativas em recursos; na determinação de estratégias para gerenciamento de recursos; na identificação de posições de desvantagem, paridade e vantagem competitiva no mercado e na avaliação do desempenho da organização;

A partir de algumas proposições encontradas na literatura, as seguintes conclusões foram obtidas neste estudo:

- o conceito de "recurso sustentável" é abstrato, pois a característica de não ser observável é inerente ao conceito (Godfrey \& Hill, 1995). Para fins gerenciais, foi proposto neste estudo que, em vez de tentar identificar recursos, os administradores devem gerar um sistema de indicadores objetivos que permita analisar o resultado do gerenciamento de recursos da organização.

- o termo "vantagem competitiva" é tautológico quando usado isoladamente (Klein, 2002). Para fazer sentido, o termo vantagem competitiva deve ser relacionado com a dinâmica competitiva e situado em termos de um par oferta/mercado. Assim, uma organização pode simultaneamente possuir uma posição de vantagem competitiva em um determinado par oferta/mercado e desvantagem competitiva em outro.

- o processo de administração estratégica está relacionado e ocorre simultaneamente com o processo de competição (Grassl, 2003). As organizações criam e modificam suas 
Vantagem Competitiva: Operacionalizando o Conceito a partir da Resource-Advantage Theory

estratégias, de forma proativa ou reativa, conforme o contexto competitivo em que estão inseridas.

- a função estratégica sob a ótica dos administradores consiste na conversão de recursos em alguma forma de valor para o consumidor (Fahy, 1999). O gerenciamento de recursos deve impactar na eficiência e/ou eficácia na geração de valor da oferta da organização. O valor da oferta irá determinar o desempenho relativo da organização no processo de competição.

- a Resource-Based Theory pode ser utilizada como base para a análise interna das empresas (Barney, 2001). A análise de posições de vantagens e desvantagens comparativas em recursos pode indicar os pontos fortes e fracos da organização e, portanto, o conceito de "recursos" pode ser utilizado como base para análise interna.

Utilizando a dinâmica de competição proposta pela RA Theory, este estudo procurou-se combinar alguns conceitos teóricos amplamente discutidos na literatura (especialmente "recursos" e “vantagem competitiva") e questionados por alguns autores do ponto de vista de aplicação prática, para geração de um framework gerencial de análise interna das organizações. O framework proposto neste artigo é inédito e deve ser aprimorado. Outros métodos de análise podem ser obtidos com novas formas de combinação dos conceitos ou com a utilização de outros conceitos. No entanto, para esta pesquisa, o importante foi a construção de formas de operacionalizar a teoria existente e coletar reflexões sobre os conceitos estudados a partir das experiências práticas vivenciadas dentro das organizações.

\section{REFERÊNCIAS}

Addis, Michela and Stefano Podesta (2005), "Long life to marketing research: a postmodern view". European Journal of Marketing, 39 (3/4), $386-412$.

http://dx.doi.org/10.1108/03090560510581836

Adner, Ron and Peter Zemsky (2006), "A demand-based perspective on sustainable competitive advantage". Strategic Management Journal, 27 (3), 215-239.

http://dx.doi.org/10.1002/smj.513

Alderson, Wroe (1937), “A marketing view of competition”. Journal of Marketing, 1 (3), 189-190. http://dx.doi.org/10.2307/1245640

Revista Ibero-Americana de Estratégia - RIAE, São Paulo, v. 8, n. 2, p. 31-57, jul./dez. 2009. 
Anderson, James C., James A. Narus and Wouter Van Rossum (2006), "Customer value propositions in business markets”. Harvard Business Review, 84 (3), 90-99.

Ardley, Barry (2008), "Marketing textbooks and the misrepresentation of reality". Qualitative Market Research: An International Journal, 11 (4), 372 - 385.

http://dx.doi.org/10.1108/13522750810901484

Bartlett, Christopher A. and Sumantra Ghoshal (2002), "Building competitive advantage through people," MIT Sloan Management Review, 43 (2), 33- 42.

Barney, Jay B (1991), "Firm resources and sustained competitive advantage," Journal of Management, 17 (1), 99-120.

http://dx.doi.org/10.1177/014920639101700108

(2001), Gaining and sustaining competitive advantage, Englewood Cliffs: Prentice Hall.

Besanko, David, David Dranove and Mark Shanley (2000), Economics of strategy, New York: John Wiley \& Sons.

Bryman, Alan (2005), Research methods and organization studies, London: Routledge.

Cardy, Robert L. and T. T. Selvarajan (2006), "Competencies: alternative frameworks for competitive advantage," Business Horizons, 49 (3), 235-245.

http://dx.doi.org/10.1016/j.bushor.2005.09.004

Castro, Julio O. de and James J. Chrisman (1995), “Order of market entry, competitive strategy, and financial performance,” Journal of Business Research, 33 (2), 165-177.

http://dx.doi.org/10.1016/0148-2963(94)00066-N

Chaharbaghi, Kazem and Richard Lynch (1999), "Sustainable competitive advantage: towards a dynamic resource-based strategy," Management Decision, 37 (1), 45-50.

http://dx.doi.org/10.1108/00251749910252012

Christensen, Clayton M. (2001), "The past and future of competitive advantage," MIT Sloan Management Review, 42 (2), 105-109.

Connor, Tom (2002), "The resource-based view of strategy and its value to practising managers," Strategic Change, 11 (6), 307-316.

http://dx.doi.org/10.1002/jsc.593

Revista Ibero-Americana de Estratégia - RIAE, São Paulo, v. 8, n. 2, p. 31-57, jul./dez. 2009. 
Cousins, Paul D. (2005), "The alignment of appropriate firm and supply strategies for competitive advantage," International Journal of Operations \& Production Management, 25 (5), 403-428.

http://dx.doi.org/10.1108/01443570510593120

Coyne, Kevin P. (1986), "Sustainable competitive advantage: what it is, what it isn't," Business Horizons, 29 (1), 54-61.

http://dx.doi.org/10.1016/0007-6813(86)90087-X

Day, George S. (1984), Strategic market planning: the pursuit of competitive advantage, St. Paul: West Publishing Company.

52.

(1994), “The capabilities of market-driven organizations,” Journal of Marketing, 58 (4), 37 http://dx.doi.org/10.2307/1251915

and Robin Wensley (1988), “Assessing advantage: a framework for diagnosing competitive superiority,” Journal of Marketing, 52 (2), 1-20.

Fahy, John (1999), "Strategic marketing and the resource-based view of the firm," Academy of Marketing Science Review, 10 (1), 1-21.

Ford, David N. and Laurent A. Mahieu (1998), "Operationalising the resource-based view of the firm,” In: International System Dynamics Conference, XVI, 1998, Quebec. Proceedings... Canada: System Dynamics Society, 1-16.

Foss, Nicolai J. (1997), "The resource-based perspective: an assessment and diagnosis of problems," DRUID (Danish Research Unit for Industrial Dynamics), 97 (1), 1-33.

Ghemawat, Panjak (1986), “Sustainable advantage,” Harvard Business Review, 64 (5), 53-58.

Godfrey, Paul C. and Charles W. L. Hill (1995), "The problem of unobservables in strategic management research,” Strategic Management Journal, 16 (7), 519-533.

http://dx.doi.org/10.1002/smj.4250160703

Grassl, Wolfgang (2003), "Review: a general theory of competition: resources, competences, productivity, economic growth," The Review of Austrian Economics, 16 (4), 385-393.

http://dx.doi.org/10.1023/A:1027353307279

Hair Jr., Joseph F., Barry Babin, Arthur H. Money and Phillip Samouel (2003), Essentials of Business Research Methods, New York: John Wiley \& Sons.

Hamel, Gary and C. K. Prahalad (1989), "Strategic intent," Harvard Business Review, 67 (3), 63 76.

Revista Ibero-Americana de Estratégia - RIAE, São Paulo, v. 8, n. 2, p. 31-57, jul./dez. 2009. 
Hayek, Friedrich A. Von (1945), "The use of knowledge in society," American Economic Review, 35 (4), 519-530.

Henderson, Bruce D. (1983), “The anatomy of competition,” Journal of Marketing, 47 (2), 7-11. http://dx.doi.org/10.2307/1251489

Hooley, Graham J., Gordon E. Greenley, John W. Cadogan and John Fahy (2005), "The performance impact of marketing resources," Journal of Business Research, 58 (1), 18 - 27. http://dx.doi.org/10.1016/S0148-2963(03)00109-7

Hunt, Shelby D. (1995), "The resource-advantage theory of competition: toward explaining productivity and economic growth," Journal of Management Inquiry, 4 (4), 317-332. http://dx.doi.org/10.1177/105649269500400403

(1997a), "Competing through relationship: grounding relationship marketing in resource advantage theory," Journal of Marketing Management, 13 (4), 431-445.

(1997b), "Evolutionary economics, endogenous growth models, and resource-advantage theory," Eastern Economic Journal, 23 (4), 425-439.

(1997c), "Resource-advantage theory: an evolutionary of competition firm behaviour?," The Journal of Economic Issues, 31 (1), 59-77.

(1997d), "Resource-advantage theory the wealth of nations," The Journal of SocioEconomics, 26 (4), 335-357.

http://dx.doi.org/10.1016/S1053-5357(97)90001-9

(1998), "Productivity, economic growth, and competition: resource allocation or resource creation," Business and the Contemporary World, 10 (3), 367-394.

(1999), "The strategic imperative and sustainable competitive advantage: public policy implications of resource-advantage theory," Journal of the Academy of Marketing Science, 27 (2), 144-159.

http://dx.doi.org/10.1177/0092070399272003

(2000a), A general theory of competition: resources, competences, productivity and economic growth, Thousand Oaks: Sage.

(2000b), "A general theory of competition: too ecletic or not ecletic enough? Too incremental or not incremental enough? Too neoclassical or not neoclassical enough?," Journal of Macromarketing, 20 (1), 77-81.

Revista Ibero-Americana de Estratégia - RIAE, São Paulo, v. 8, n. 2, p. 31-57, jul./dez. 2009. 
Vantagem Competitiva: Operacionalizando o Conceito a partir da Resource-Advantage Theory

(2001), "A general theory of competition: issues, answers and an invitation," European Journal of Marketing, 35 (5/6), 524-548.

http://dx.doi.org/10.1108/03090560110388097

G.

(2002), "Resource-advantage theory and Austrian economics," In. Nicolai J. Foss and Peter

Klein, (ed.), Entrepreneurship and the firm: Austrian perspectives on economic organization, Aldershot: Edward Elgar Publishing, 266-286.

and Dennis B. Arnett (2001), "Competition as an evolutionary process and antitrust policy," Journal of Public Policy \& Marketing, 20 (1), 15-26.

http://dx.doi.org/10.1509/jppm.20.1.15.17296

and

(2004), "Market segmentation strategy, competitive advantage, and public policy: grounding segmentation strategy in resource-advantage theory," Australasian Marketing Journal, 12 (1), 7-25.

http://dx.doi.org/10.1016/S1441-3582(04)70083-X

and Caroline Derozier (2004), "The normative imperatives of business and marketing strategy: grounding strategy in resource-advantage theory," Journal of Business \& Industrial Marketing, 19 (1), 5-22.

http://dx.doi.org/10.1108/08858620410516709

and Dale F. Duhan (2002), "Competition in the third millennium: efficiency or effectiveness?,” Journal of Business Research, 55 (2), 97-102.

http://dx.doi.org/10.1016/S0148-2963(00)00144-2

and C. Jay Lambe (2000), "Marketing's contribution to business strategy: market orientation, relationship marketing and resource-advantage theory," International Journal of Marketing Reviews, 2 (1), 17-43.

and Robert M. Morgan (1995), "The comparative advantage theory of competition," Journal of Marketing, 59 (2), 1-14.

http://dx.doi.org/10.2307/1252069

and

(1996), "The resource-advantage theory of competition: dynamics, path dependencies, and evolutionary dimensions," Journal of Marketing, 60 (4), 107-114.

http://dx.doi.org/10.2307/1251905

and

(1997), "Resource-advantage theory: a snake swallowing its tail or a general theory of competition?," Journal of Marketing, 61 (4), 74-82.

http://dx.doi.org/10.2307/1252088

Revista Ibero-Americana de Estratégia - RIAE, São Paulo, v. 8, n. 2, p. 31-57, jul./dez. 2009. 
Klein, Jeremy (2002), “Beyond competitive advantage,” Strategic Change, 11 (6), 317-327. http://dx.doi.org/10.1002/jsc.606

Kothandaraman, Prabakar and David T. Wilson (2001), "The future of competition: value-creating networks," Industrial Marketing Management, 30 (4), 379-389.

http://dx.doi.org/10.1016/S0019-8501(00)00152-8

Lei, David and John W. Jr. Slocum (2002), “Organization designs to renew competitive advantage," Organizational Dynamics, 31 (1), 1-18.

http://dx.doi.org/10.1016/S0090-2616(02)00068-2

Lieberman, Marvin B. and Shigeru Asaba (2006), "Why do firms imitate each other?," Academy of Management Review, 31 (2), 366-385.

http://dx.doi.org/10.5465/AMR.2006.20208686

Littler, Dale and Caroline Tynan (2005), "Where are we and where are we going? The status and future of research in marketing," European Journal of Marketing. 39 (3/4), 261 - 271.

http://dx.doi.org/10.1108/03090560510581764

Lubit, Roy (2001), "Tacit knowledge and knowledge management: the keys to sustainable competitive advantage," Organizational Dynamics, 29 (4), 164-178.

http://dx.doi.org/10.1016/S0090-2616(01)00026-2

Ma, Hao (1999a), "Anatomy of competitive advantage: a SELECT framework," Management Decision, 37 (9), 709-718.

http://dx.doi.org/10.1108/00251749910299129

(1999b), "Creation and preemption for competitive advantage," Management Decision, 37 (3), 259-266.

http://dx.doi.org/10.1108/00251749910264497

Morgan, Neil A., Douglas W. Vorhies and Bodo B. Schlegelmilch (2006), "Resource-performance relationships in industrial export ventures: the role of resource inimitability and substitutability," Industrial Marketing Management, 35 (5), 621-633.

http://dx.doi.org/10.1016/j.indmarman.2005.05.018

Morgan, Robert E. and Shelby D. Hunt (2002), "Determining marketing strategy: a cybernetic systems approach to scenario planning," European Journal of Marketing, 36 (4), 450-478.

http://dx.doi.org/10.1108/03090560210417264

Revista Ibero-Americana de Estratégia - RIAE, São Paulo, v. 8, n. 2, p. 31-57, jul./dez. 2009. 
Vantagem Competitiva: Operacionalizando o Conceito a partir da Resource-Advantage Theory

Oliver, Christine (1997), "Sustainable competitive advantage: combining institutional and resourcebased view," Strategic Management Journal, 18 (9), 697-713.

http://dx.doi.org/10.1002/(SICI)1097-0266(199710)18:9<697::AID-SMJ909>3.0.CO;2-C

Olusoga, S. Ade, Michael P. Mokwa and Charles H. Noble (1995), "Strategic groups, mobility barriers, and competitive advantage: an empirical investigation," Journal of Business Research, 33 (2), 153-164.

http://dx.doi.org/10.1016/0148-2963(94)00065-M

Peteraf, Margaret A. (1993), "The cornerstones of competitive advantage: a resource-based view," Strategic Management Journal, 14 (3), 179-191.

http://dx.doi.org/10.1002/smj.4250140303

Porter, Michael E. (1980), Competitive strategy, New York; Free Press.

(1985), Competitive advantage, New York: Free Press.

Reed, Richard and Robert J. DeFillippi (1990), "Causal ambiguity, barriers to imitation, and sustainable competitive advantage,” Academy of Management Review, 15 (1), 88-102.

http://dx.doi.org/10.5465/AMR.1990.4308277

http://dx.doi.org/10.2307/258107

Rindova, Violina P. and Charles J. Fombrun (1999), "Constructing competitive advantage: the role of firm-constituent interactions," Strategic Management Journal, 20 (8), 691-710.

http://dx.doi.org/10.1002/(SICI)1097-0266(199908)20:8<691::AID-SMJ48>3.0.CO;2-1

Rowe, W. Glenn and James G. Barnes (1998), "Relationship marketing and sustained competitive advantage," Journal of Market Focused Management, 2 (3), 281-297.

http://dx.doi.org/10.1023/A:1009707818053

Rouse, Michael J. and Urs S. Daellenbach (1999), "Rethink research methods for the resourcebased perspective: isolating sources of sustainable competitive advantage," Strategic Management Journal, 20 (5), 487-494.

http://dx.doi.org/10.1002/(SICI)1097-0266(199905)20:5<487::AID-SMJ26>3.0.CO;2-K

Voss, Chris, Nikos Tsikriktsis and Mark Frohlich (2002), "Case research in operations management," International Journal of Operations \& Production Management, 22 (2), 195-219. http://dx.doi.org/10.1108/01443570210414329

Weerawardena, Jay and Aron O'Cass (2004), "Exploring the characteristics of the market-driven firms and antecedents to sustained competitive advantage," Industrial Marketing Management, 33 (5), 419-428.

http://dx.doi.org/10.1016/j.indmarman.2003.07.002

Revista Ibero-Americana de Estratégia - RIAE, São Paulo, v. 8, n. 2, p. 31-57, jul./dez. 2009. 
Wright, Peter, Mark J. Kroll, Bevalee Pray and Augustine Lado (1995), "Strategic orientations, competitive advantage, and business performance," Journal of Business Research, 33 (2), 143151.

http://dx.doi.org/10.1016/0148-2963(94)00064-L

Yin, Robert K. (2002), Case study research: design and methods, Thousand Oaks: Sage.

Recebido: 01/08/09

Aprovado: 29/10/09

Revista Ibero-Americana de Estratégia - RIAE, São Paulo, v. 8, n. 2, p. 31-57, jul./dez. 2009. 